\title{
Keynote Talk
}

\section{Broader Impacts: Research You Can Use}

\author{
Judith Olson \\ Don Bren School of Information and Computer Sciences \\ University of California, Irvine \\ Irvine, CA, USA \\ jsolson@uci.edu
}

\begin{abstract}
A number of threads of thought have come together recently having to do with how we make our research useable and useful to the world. One thread is inspired by a movement in medicine called Clinical Translational Science in which funding is given to researchers to translate basic research into guidelines, treatments, and regimens that clinicians can use. A second thread arose in reflecting about our own recent work in which we translated a theory about what makes for good distance collaboration into an online assessment tool and administered it to hundreds of people involved in remote collaboration. Upon completion of the assessment, each participant immediately gets a personalized report on the strengths of their collaboration, the challenges, and what to do about it. We get the data, and they get the help. These two threads point to making a difference, having broader impact.
\end{abstract}

In this talk I will review some ways we can have an impact, both directly to people, through design practice (our clinicians), and via a myriad of other tools while doing good research. I encourage us all to spend more energy on having more direct effects on the world in which we live.

Categories \& Subject Descriptors: H.1.2 [Models and Principles]: User/Machine Systems human factors; H.5.3. [Information Interfaces and Presentation]: Group and Organization Interfaces - asynchronous interaction, collaborative computing; K.4.3. [Computers and Society]: Organizational Impacts - computer-supported cooperative work

General Terms: Design, Experimentation, Human Factors.

\section{Bio}

Judith Olson is the Bren Professor of Information and Computer Sciences in the Informatics Department at the UC Irvine, with courtesy appointments in the School of Social Ecology and the Merage School of Business. For 20 years, she has focused on distributed teams, summaries of which are found in her most cited paper, "Distance Matters," (Olson \& Olson, 2000), in her key theoretical contribution in the book Scientific Collaboration on the Internet, and her upcoming book, Working Together Apart. She has studied distributed teams both in the field and in the laboratory, finding the myriad of communication hurdles distributed teams have, the consequent underutilization of remote team members' skills and reduction in trust. Her current work includes studying two of the most difficult aspects of long distance collaboration: Crossing cultures and having no overlap in workdays. She is an ACM Fellow, was in the first class of inductees in CHI's Academy, holds a Lifetime Achievement award from SIGCHI, and was awarded the 2011 ACM Athena Award, translating to "woman of the year in computing."

Copyright is held by the author/owner(s).

CSCW'12, February 11-15, 2012, Seattle, Washington, USA.

ACM 978-1-4503-1086-4/12/02. 